\title{
The Prevalence and Risk Factors of Cerebral Microbleeds: A Community-Based Study in China
}

This article was published in the following Dove Press journal:

Therapeutics and Clinical Risk Management

\author{
Qi Luo $\mathbb{D}^{1, *}$ \\ Huidong Tang ${ }^{1} *$ \\ Xinxin $\mathrm{Xu}^{2}$ \\ Juan Huang ${ }^{2}$ \\ Pei Wang' \\ Guiying $\mathrm{He}^{\prime}$ \\ Xiaoxuan Song' \\ Yumeng Huang' \\ Shengdi Chen' \\ Fuhua Yan $^{2}$ \\ Yuyan Tan' \\ Jianfang $\mathrm{Ma}^{\prime}$ \\ 'Department of Neurology, Ruijin \\ Hospital Affiliated to Shanghai Jiao Tong \\ University School of Medicine, Shanghai, \\ People's Republic of China; ${ }^{2}$ Department \\ of Radiology, Ruijin Hospital Affiliated to \\ Shanghai Jiao Tong University School of \\ Medicine, Shanghai, People's Republic of \\ China
}

*These authors contributed equally to this work
Correspondence: Jianfang Ma

Department of Neurology, Ruijin Hospital Affiliated to Shanghai Jiao Tong University School of Medicine, No. 197, Second Ruijin Road, Shanghai, 200025, People's Republic of China

$\mathrm{Tel} / \mathrm{Fax}+86$ 2I-6445-4473

Email mjfl0924@rjh.com.cn
Introduction: Cerebral microbleeds (CMBs) are frequently found in the healthy elderly. However, data on the prevalence and risk factors of CMBs in the general population of China are lacking.

Methods: A cross-sectional study focusing on the prevalence and risk factors of CMBs was conducted in stroke-free elderly from Shanghai Wuliqiao community. MRI was performed at 3Tesla and cardiovascular risk factors (eg, age, smoking history, and hypertension), cerebral small vessel disease (CSVD) markers (eg, white matter hyperintensities, lacunar infarction, and enlarged perivascular space) and genetic information (eg, APOE, CR1) were recorded. Poisson regression was used to analyze the risk factors of the presence and location of microbleeds.

Results: A total of 199 participants (70.8 \pm 7.2 years old; male $31.2 \%)$ were finally included in our analysis. The overall prevalence of CMBs was $12.6 \%(25 / 199)$ and increased with age from $7.5 \%$ (55-64 years old) to $19.3 \%$ (over 75 years old). Of those with CMBs, most of them (16/25) located in the deep/mixed region and had 1-2 CMBs (18/25). Poisson regression analysis showed that white matter hyperintensities (OR=1.22, 95\% CI: 1.16-1.29), APOE $\varepsilon 4+$ carrier $(\mathrm{OR}=2.16,95 \% \mathrm{CI}: 1.18-3.96)$ and $\mathrm{CR} 1$ non-F/F isoform $(\mathrm{OR}=7.78$, 95\% CI: 4.34-13.96) were associated with CMBs. Further analysis found that in addition to the above three risk factors, hypertension $(\mathrm{OR}=2.98,95 \% \mathrm{CI}: 1.16-7.64)$, lacunar infarction $(\mathrm{OR}=2.39,95 \% \mathrm{CI}: 1.19-4.81)$ also increased the risk of deep/mixed CMBs.

Conclusion: The prevalence of cerebral microbleeds is similar to other countries. Cardiovascular risk factors, CSVD markers, and genetic factors (APOE $\varepsilon 4, \mathrm{CR} 1$ non-F/F isoform) were associated with CMBs, suggesting an interaction of multiple pathogenesis in Chinese stroke-free community population.

Keywords: cerebral microbleeds, prevalence, risk factors, community-based study

\section{Introduction}

Cerebral microbleeds (CMBs) are $2-10 \mathrm{~mm}$, rounded or circular, well-defined hypointense lesions on gradient-echo T2*-weighted images (GRE T2*WI) or susceptibilityweighted images (SWI) of magnetic resonance imaging (MRI). ${ }^{1}$ As one feature of cerebral small vessel disease (CSVD), CMBs were found to have a close association with ischemic or hemorrhagic stroke, dementia, or even mortality. ${ }^{2}$

The prevalence of CMBs in the normal elderly varies across different studies, ranging from $4.0 \%{ }^{3}$ to $26.9 \%{ }^{4}$ However, limited studies have been done in China. There was one study done in Shanghai urban area that reported a prevalence of $7.3 \%,{ }^{5}$ but with $1.5 \mathrm{~T}$ scanner, which may reduce the detection of CMBs. Another study was done on rural areas and showed the prevalence of $10.6 \%{ }^{6}$ 
The etiology of CMBs is complex. Many studies suggested that deep CMBs may relate to hypertensive small vessel disease (HTN-SVD) and strictly lobar CMBs for cerebral amyloid angiopathy (CAA), ${ }^{1}$ but the risk factors of CMBs are not entirely clear. Previous studies found APOE genotype, cardiovascular factors, and CSVD were associated with CMBs. ${ }^{7}$ With the different distribution of CMBs between Western and Eastern countries, ${ }^{3}$ it remains unknown whether there are also different risk factors of CMBs either. Studies in Japan found hypertension and lower serum total cholesterol were associated with deep/ infratentorial CMBs risk, while APOE $\varepsilon 4$ was related to lobar CMBs. ${ }^{8}$ Other Asian countries like Singapore reported age and hypertension were risk factors of CSVD. ${ }^{4}$ Few studies focused on the risk factors of CMBs in Chinese population. Only one study of 1211 individuals in Shunyi city confirmed age and hypertension increased the risk of deep CMBs. However, this study did not have CSVD evaluation and genetic investigation. ${ }^{6}$

Hence, we performed a cross-sectional study with $3 \mathrm{~T}$ MRI to investigate the prevalence and risk factors of CMBs, including cardiovascular factors (eg, hypertension, diabetes), CSVD evaluation (eg, white matter hyperintensity, lacunar infarction, and enlarged perivascular space) and genetic investigation (eg, APOE, CR1), in a strokefree Chinese community cohort.

\section{Method}

\section{Study Participants}

The Wuliqiao cohort is an ongoing longitudinal community-based study designed to assess risk factors of neurodegenerative diseases including dementia and Parkinson's disease. The inclusion criteria for Wuliqiao cohort: A) aged $\geq 50$ years; B) willing to participate. Three thousand nine hundred and seventy inhabitants were recruited in Wuliqiao community (urban) of Shanghai at baseline (2009-2011) and followed up in 2014-2015 and 2018-2019.9,10

Participants in this study (MRI sub-study) were enrolled during the second follow-up 2018-2019 period. Inclusion criteria included: A) willing to participate; B) no MRI contraindications: implantable devices, intraorbital metallic foreign body, intracranial aneurysm clips, cardiac pacemaker, valvular prosthesis, and cochlear implants; C) free of stroke: individuals without a history of symptomatic ischemic stroke and intracerebral hemorrhage (ICH), which should be confirmed by CT or MRI; D) free of other neurological diseases including major head trauma, brain surgery, brain tumor, psychiatric diseases, Parkinson's disease and dementia. Two hundred and nine individuals were enrolled in which 10 individuals were excluded due to claustrophobia $(n=6)$ and poor image pictures $(n=4)$. Therefore, a total of 199 individuals with eligible data were included in the final analysis.

\section{Standard Protocol Approval, Registration, and Patient Consent}

The study was approved by Ethics Committee of Ruijin Hospital affiliated to Shanghai Jiao Tong University School of Medicine, China. Written informed consent was obtained from each participant of this study.

\section{Cardiovascular Risk Factors}

Cardiovascular risk factors were collected at the time of MRI by interview, laboratory, or physical examination. Demographic information including age at MRI, sex, height and weight, and smoking history were documented. Related diseases, including hypertension, diabetes, and atrial fibrillation, and medicine history, including antithrombotics (antiplatelets and anticoagulants) and statins, were also documented (See supplemental data).

\section{Magnetic Resonance Imaging Parameters and CSVD Evaluations}

All subjects were scanned on 3T Philips Ingenia (Philips Healthcare, Best, The Netherlands) at the Radiology Department of Ruijin Hospital. Susceptibility-weighted images (SWI; repetition time (TR) $=25 \mathrm{~ms}$, echo time $(\mathrm{TE})=18 \mathrm{~ms}$, field of view $(\mathrm{FOV})=256 \times 256 \mathrm{~mm}^{2}, 54$ slices, 2-mm slice thickness) were acquired in axial plane. Three-dimensional (3D) T1-weighted images (T1WI; $\mathrm{TE}=7.5 \mathrm{~ms}, \mathrm{TR}=25 \mathrm{~ms}, 64$ slices, $2-\mathrm{mm}$ slice thickness) and fluid-attenuated inversion recovery, spectral presaturation with inversion recovery (Flair-SPIR; TE $=120 \mathrm{~ms}$, $\mathrm{TR}=9000 \mathrm{~ms}, 32$ slices, $3-\mathrm{mm}$ slice thickness) were also acquired. All CSVD evaluation (including CMBs) was done by two experienced radiologists (See supplemental data).

Microbleed anatomical rating scale (MARS) was used to evaluate the number and location of CMBs. ${ }^{11}$ Lobar region includes cortical and subcortical regions with U fibers. Basal ganglia, thalamus, internal capsule, external capsule, corpus callosum, and deep and periventricular white matter belong to the deep region, and the 
infratentorial region is composed of brainstem and cerebellum. Scale of age-related white matter changes (ARWMC) was used as a general measure of white matter hyperintensities. $^{12}$ White matter changes on MRI were defined as bright lesions $\geq 5 \mathrm{~mm}$ on FLAIR images. Lacune was rated visually as a deep, focal lesion (3-15 mm), hypointense in 3D T1WI and had a hyperintense rim surrounding the lesions in FLAIR sequence. ${ }^{13}$ Enlarged perivascular space (EPVS) was defined as visible small dots or linear structures of hypointensity $(\leq 3 \mathrm{~mm})$ in $3 \mathrm{D}$ T1WI and FLAIR. ${ }^{14}$ EPVS was evaluated in basal ganglia and centrum semiovale, respectively.

\section{Apolipoprotein E Genotyping and CR I Isoforms Test}

Apolipoprotein E (APOE) genotyping was performed by company (MAP Biotech, Shanghai). APOE genotyping was available for 196 participants (98.5\%). The genotype and allele frequencies of rs429358 and rs7412 in our population were in Hardy-Weinberg equilibrium.

Complement $\mathrm{C} 3 \mathrm{~b} / \mathrm{C} 4 \mathrm{~b}$ receptor 1 (CR1) isoforms were determined by Western blot and paralogue ratio test (PRT) tests (See supplemental data). The different isoforms of CR1 reflect the copy number variations, and $\mathrm{F}$ isoform $(190 \mathrm{kDa})$ reflects 2 copy numbers, $\mathrm{S}$ isoform $(220 \mathrm{kDa})$ reflects 3 copy numbers and the rare $F^{\prime}$ isoform $(160 \mathrm{kDa})$ with 1 copy number. ${ }^{15}$ Most individuals carried $\mathrm{F} / \mathrm{F}$ isoform and few $\mathrm{CR} 1$ non-F/F isoform, including $11 \mathrm{~F} / \mathrm{S}$ isoform, $2 \mathrm{~S} / \mathrm{S}$ isoform, and $3 \mathrm{~F} / \mathrm{F}^{\prime}$ isoform (See supplemental data).

\section{Statistics}

Of those with CMBs, two groups were divided according to the previous studies. ${ }^{7}$ One was individuals with CMBs restricted to a lobar region, called strictly lobar $\mathrm{CMBs}$, and the other was individuals with CMBs in a deep region, with or without lobar CMBs, called deep/mixed CMBs. Distribution of cerebellar microbleed may be related with different pathogenic mechanism, ${ }^{16}$ and we did not include 1 individual with strictly cerebellar microbleed into the group of deep/mixed CMBs. CR1 was divided into two groups, including $\mathrm{F} / \mathrm{F}$ isoform and non- $\mathrm{F} / \mathrm{F}$ isoform $(\mathrm{F} / \mathrm{S}$, $\mathrm{S} / \mathrm{S}, \mathrm{F} / \mathrm{F}$ ' isoform). Poisson regression, which makes full use of the information about the number of CMBs, was used to analyze the risk factors of CMBs. Model 1 adjusted for age and sex and Model 2 adjusted for all positive factors in Model 1. Categorical variables are presented as count (percentage, \%) and continuous variables as mean (standard deviation, SD) or median (interquartile range, IQR). $\mathrm{P}$ value $<0.05$ was considered statistically significant. All analyses were performed by SPSS 25.0.

\section{Results}

Total of 199 participants were included in this study. Mean age was 70.8 years old and $62(31.2 \%)$ were male. The demographic data on brain MRI variables and genetic information were shown in Table 1 . The prevalence of overall CMBs was $12.6 \%$ and increased along with age (Table 2). Of those with CMBs $(n=25), 8$ individuals had strictly lobar CMBs (only 2 with multiple $(n \geq 2)$ strictly lobar CMBs), 16 had deep/mixed CMBs (8 strictly deep CMBs and $8 \mathrm{CMBs}$ in both lobar and deep regions), and 1 had strictly infratentorial CMBs.

We conducted Poisson regression analysis to investigate the risk factors of CMBs (Table 3). Due to the limited sample size, analyses were performed in all CMBs and deep/mixed CMBs, but not strictly lobar CMBs. APOE $\varepsilon 4$ + carriers $(\mathrm{OR}=2.16,95 \% \mathrm{CI}: 1.18-3.96), \mathrm{CR} 1$ non-F/F isoform $(\mathrm{OR}=7.78,95 \% \mathrm{CI}$ : 4.34-13.96) and white matter hyperintensities $(\mathrm{OR}=1.22$, 95\% CI: 1.16-1.29) increased the risk of all CMBs. However, diabetes $(\mathrm{OR}=0.24,95 \%$

Table I Demographic and Clinical Characteristics of Participants

\begin{tabular}{|c|c|}
\hline & All $(n=199)$ \\
\hline Age at MRI, y, mean (SD) & $70.8(7.2)$ \\
\hline Sex, male, n (\%) & $62(31.2)$ \\
\hline Obesity $^{\mathrm{a}}$, n (\%) & $18(9.0)$ \\
\hline Smoking history, n (\%) & $36(18.1)$ \\
\hline Hypertension, n (\%) & 91 (45.7) \\
\hline Diabetes, n (\%) & $28(14.1)$ \\
\hline Atrial fibrillation, $\mathrm{n}(\%)$ & $5(2.5)$ \\
\hline Antithrombotics, n (\%) & $70(35.2)$ \\
\hline Statin, $n(\%)$ & $32(16.1)$ \\
\hline Microbleeds, n(\%) & $25(12.6)$ \\
\hline ARWMC, Median (IQR) ${ }^{b}$ & $5(3,8)$ \\
\hline Lacunar Infarction, n (\%) ${ }^{b}$ & $33(16.8)$ \\
\hline EPVS, $n(\%){ }^{b, c}$ & $16(8.1)$ \\
\hline APOE $\varepsilon 4+$ carrier, $n(\%){ }^{d}$ & $39(19.9)$ \\
\hline CRI non-F/F isoform, $n(\%)^{e}$ & $16(8.0)$ \\
\hline
\end{tabular}

Notes: a Obesity was considered when BMI $\geq 28 \mathrm{~kg} / \mathrm{m}^{2 ;}$ b Two individuals fail to complete the FLAIR sequence; ${ }^{c} 15$ individuals with basal ganglion-EPVS predominated and $\mathrm{I}$ individuals with equal degree; ${ }^{\mathrm{d}}$ Three individuals did not give consent for genotyping; ${ }^{\mathrm{e}} \mathrm{CRI}$ non-F/F isoform includes II F/S isoform, $2 \mathrm{~S} / \mathrm{S}$ isoform, and 3 F/F' isoform.

Abbreviations: CMBs, cerebral microbleeds; MRI, magnetic resonance imaging; ARWMC, age-related white matter changes; EPVS, enlarged perivascular space; APOE, apolipoprotein E; CRI, complement $\mathrm{C} 3 \mathrm{~b} / \mathrm{C} 4 \mathrm{~b}$ receptor I; SD, standard deviation; IQR, interquartile range. 
Table 2 Age-Specific Prevalence of CMBs

\begin{tabular}{|c|c|c|c|c|}
\hline Age Range & Participants, $n$ & CMB, \% (n) & Multiple ( $\geq 2)$ CMBs, \% (n) & Strictly Lobar CMBs, \% (n) \\
\hline $55-64$ y & 40 & $7.5(3)$ & $2.5(I)$ & $2.5(1)$ \\
\hline $65-74$ y & 102 & $10.8(11)$ & $4.9(5)$ & $3.9(4)$ \\
\hline $75-92$ y & 57 & $19.3(11)$ & $10.5(6)$ & $5.3(3)$ \\
\hline
\end{tabular}

Abbreviation: CMBs, cerebral microbleeds.

Table 3 Risk Factors of CMBs by Poisson Regression

\begin{tabular}{|c|c|c|c|c|}
\hline \multirow[t]{2}{*}{ Variables } & \multicolumn{2}{|c|}{ Overall CMB vs No CMB, OR $(95 \% \mathrm{Cl})^{\mathrm{h}}$} & \multicolumn{2}{|c|}{ Deep/Mixed vs No CMB, OR $(95 \% \mathrm{Cl})$} \\
\hline & Model I & Model 2 & Model I & Model 2 \\
\hline Age ${ }^{a}$, per year & $1.07(1.03,1.10)$ & $1.02(0.98,1.06)$ & $1.06(1.02,1.09)$ & $0.99(0.93,1.05)$ \\
\hline Sex ${ }^{b}$, male, vs female & $2.16(1.39,3.38)$ & $0.83(0.33,2.05)$ & $3.39(2.00,5.76)$ & I. $17(0.37,3.70)$ \\
\hline Obesity $^{c}$ & $3.24(1.96,5.34)$ & I.II $(0.52,2.40)$ & $4.45(2.6 I, 7.6 I)$ & $0.70(0.26,1.93)$ \\
\hline Smoking history & $3.80(1.96,7.40)$ & $\mathrm{I} .43(0.55,3.70)$ & $4.66(2.22,9.78)$ & $0.99(0.29,3.40)$ \\
\hline Hypertension & $2.18(1.35,3.24)$ & $1.64(0.92,2.92)$ & $4.52(2.35,8.69)$ & $2.98(1.16,7.64)$ \\
\hline Diabetes & $0.17(0.05,0.55)$ & $0.24(0.07,0.77)$ & $0.14(0.03,0.57)$ & $0.23(0.05,1.06)$ \\
\hline Atrial fibrillation & $2.02(0.63,6.49)$ & - & $2.81(0.86,9.16)$ & - \\
\hline Antithrombotics & $0.39(0.23,0.68)$ & $0.69(0.38,1.26)$ & $0.49(0.27,0.89)$ & I.07 $(0.5 \mathrm{I}, 2.24)$ \\
\hline Statin & $0.85(0.47,1.56)$ & - & $1.19(0.63,2.23)$ & - \\
\hline ARWMC $^{d}$ & $1.25(1.19,1.31)$ & $1.22(1.16,1.29)$ & $1.33(1.26,1.4 I)$ & $1.38(1.27,1.49)$ \\
\hline Lacunar Infarction ${ }^{d}$ & $1.64(1.00,2.7 I)$ & $1.29(0.76,2.19)$ & $2.11(1.22,3.64)$ & $2.39(1.19,4.81)$ \\
\hline EPVS ${ }^{\mathrm{d}, \mathrm{e}}$ & $1.57(0.81,3.05)$ & - & I.9I $(0.94,3.87)$ & - \\
\hline APOE $\varepsilon 4+$ carrier $^{f}$ & $3.16(2.00,4.99)$ & $2.16(1.18,3.96)$ & $4.33(2.60,7.20)$ & $3.72(1.60,8.65)$ \\
\hline CRI non-F/F ${ }^{g}$ & $7.26(4.62,1 \mathrm{I} .42)$ & $7.78(4.34,13.96)$ & $10.85(6.47,18.21)$ & $23.97(10.56,54.42)$ \\
\hline
\end{tabular}

Notes: Model I: adjusted for age and sex. Model 2: adjusted for all positive predictors in Model I. Bold text represents statistically significant ( $P<0.05)$; ${ }^{a}$ adjusted for sex, badjusted for age; 'Obesity was considered when BMI $\geq 28 \mathrm{~kg} / \mathrm{m}^{2 ;}{ }^{\mathrm{d}}$ Two individuals fail to complete the FLAIR sequence; ${ }^{\mathrm{e}} \mathrm{I} 5$ individuals with basal ganglion-EPVS predominated and I individuals with equal degree. ${ }^{\mathrm{f}}$ Three individuals did not give consent for genotyping; ${ }^{\mathrm{g}} \mathrm{CRI}$ non-F/F isoform includes II F/S isoform, $2 \mathrm{~S} / \mathrm{S}$ isoform, and 3 F/F' isoform; 'Further analyses on Strictly lobar vs no CMB was ceased due to poor goodness-of-fit.

Abbreviations: CMBs, cerebral microbleeds; MRI, magnetic resonance imaging; ARWMC, age-related white matter changes; EPVS, enlarged perivascular space; APOE, apolipoprotein E; CRI, complement C3b/C4b receptor I; OR, odds ratio; $95 \% \mathrm{Cl}, 95 \%$ confidence interval.

CI: 0.07-0.77) reduced the risk of all CMBs in Model 2. Other risk factors including age, male sex, obesity, smoking history, hypertension, antithrombotics, and lacunar infarction found in Model 1 did not remain statistically significant in Model 2.

Risk factors for deep/mixed CMBs were partially similar to the results of overall CMBs. The difference was that hypertension $(\mathrm{OR}=2.98,95 \% \mathrm{CI}: 1.16-7.64)$ and lacunar infarction ( $\mathrm{OR}=2.39$, 95\% CI: 1.19-4.81) were also associated with deep/mixed CMBs in Model 2 (Table 3).

\section{Discussion}

In our cross-sectional study, we found that the prevalence of CMBs in stroke-free community elderly was $12.6 \%$ and increased with age, similar to $10.6 \%$ in Shunyi study ${ }^{6}$ and $15.3 \%$ in Rotterdam Scan Study. ${ }^{17}$ To reduce confounding factors, the target populations in our study are stroke-free and non-demented elderly, representing a comparatively natural aging situation. In this cohort, we found a higher prevalence of deep/mixed CMBs than strictly lobar CMBs, supporting the findings of more deep/mixed CMBs distribution in East than West. ${ }^{3}$ And this feature prevents us from further analysis of risk factors in strictly lobar CMBs due to the limited sample.

The most interesting finding of our study was that we found two risk factors, APOE $\varepsilon 4$ and $\mathrm{CR} 1$ non-F/F isoforms $(\mathrm{F} / \mathrm{S}, \mathrm{S} / \mathrm{S}$ and $\mathrm{F} / \mathrm{F}$ ' isoforms) were associated with all CMBs and deep/mixed CMBs. APOE $\varepsilon 4$, enhancing the deposition of amyloid and more frequently in patients with $\mathrm{CAA},{ }^{18}$ has been widely reported the relationship with CMBs, usually strictly lobar CMBs. ${ }^{7,8} \mathrm{CR} 1$ has been found to increase the risk of late-onset Alzheimer's disease $(\mathrm{AD})$ and $\mathrm{CAA}^{15,18}$ and it was reasonable to hypothesize that the association of CR1 isoforms was probable more prominent with strictly lobar CMBs. But more than one study found inconsistent results on the 
relationship between APOE/CR1 and CMBs. Caunca et al showed no statistical difference in $\mathrm{CMB}$ distribution across APOE genotype. ${ }^{19} \mathrm{Li}$ et al found that APOE $\varepsilon 4 / \varepsilon 4$ genotype was a predictor for progression of cerebral microbleeds, regardless of location. ${ }^{20}$ What is more, CR1 polymorphism increased cardiovascular risks, ${ }^{21,22}$ which implies the relationship with deep/mixed CMBs.

We think that lipid metabolism and inflammation (systemic or vascular) might explain the association between APOE/CR1 and deep/mixed CMBs. Dyslipidemia is a known risk factor for cardiovascular diseases, including atherosclerosis (AS). AS may lead to $\mathrm{CMBs}$, especially in deep region, ${ }^{23}$ during the formation of collateral compensation in neovascularization. ApoE4, one lipid transporter preferring to binding large $(30-80 \mathrm{~nm})$, triglyceride-rich very low density lipoprotein (VLDL), is associated with elevated low density lipoprotein (LDL) levels and increases the risk for atherosclerosis. ${ }^{24}$ Recent studies found that CR1 on erythrocytes may be involved in the clearance of atherogenic apolipoprotein B-containing lipoprotein, like LDL. ${ }^{25}$

In addition, inflammation has a close relationship with small vessel diseases, including CMBs. ${ }^{26}$ It was shown that vascular inflammation was more associated with deep/mixed CMBs and systemic inflammation for lobar $\mathrm{CMBs}^{26}$ However, it is sometimes very difficult to ascertain whether both types of inflammation are interacted with each other. For example, long-term or severe systemic inflammation may also contribute to vascular inflammation or vice versa. APOE $\varepsilon 4$ has been found to increase inflammation by accelerating endoplasmic reticulum stress and reducing macrophage function. ${ }^{27}$ Also, APOE $\varepsilon 4$ carriers were tended to produce a stronger neuroinflammatory responding to peripheral systemic inflammation. ${ }^{28} \mathrm{CR} 1$, a receptor for complement $\mathrm{C} 3 \mathrm{~b} /$ $\mathrm{C} 4 \mathrm{~b}$, is also a central or peripheral factor involving complement mediated inflammation. Most $(\sim 80 \%) \mathrm{CR} 1$ is expressed on erythrocyte, which implies the stronger immunoregulation function in peripheral circulation. CR1 is also expressed on vascular endothelial cells, ${ }^{29}$ which may be involved in the induction and progression of atherosclerosis by influencing the assembly of the terminal complement C5b-9 complex. ${ }^{30}$ This systemic or vascular inflammation or immunoregulation function of APOE/ CR1 may explain the relationship with deep/mixed CMBs.

Cerebral small vessel disease has heterogeneous manifestations, including an ischemic phenotype (lacunar infarction, white matter hyperintensities) and a hemorrhagic one (microbleeds). ${ }^{31}$ We found that white matter hyperintensities increased the risk of all CMBs and deep/mixed CMBs, and lacunar infarction was only associated with deep/mixed CMBs. Consistently, Rotterdam Scan Study ${ }^{17}$ found that both white matter lesions and lacunar infarcts increased the risk of CMBs, especially deep or infratentorial microbleeds. The close relationship between ischemic lesions and hemorrhagic tendency in brain parenchyma may be due to the interconnected networks formed by brain microvasculature. ${ }^{31}$

Diabetes may lead to microstructural changes and one study reported the association with multiple $(\geq 2) \mathrm{CMBs}^{32}$ However, we observed the protective effect for overall CMBs, which was hard to explain and need further study. One study focusing on the elderly communities found diabetes was the protective factor of strictly lobar $\mathrm{CMBs}(\mathrm{OR}=0.69$, 95\% CI: $0.41-1.17)$, but no statistical significance $(p=0.17){ }^{8}$ Another study found the protective effect of diabetes medication. ${ }^{19}$

Although the prevalence of CMBs increased with age in our study, we did not find the association of age with CMBs in Model 2, which might be due to small sample size or counteraction by white matter changes and lacunar infarctions. We confirmed the association between hypertension and deep/ mixed CMBs, consistent with many other studies. ${ }^{8}$ We also found the protective effect of antithrombotics (mainly aspirin) in Model 1 but did not remain significant in Model 2. Several studies showed that antithrombotics did no harm in individuals with less than five CMBs. ${ }^{33}$ However, long-term use of aspirin or clopidogrel also increased the risk of CMBs. ${ }^{34}$ It remained controversial for how much risk of CMBs contributed to therapy-induced cerebral hemorrhage. We did not observe the association of statin use with $\mathrm{CMB}$ presence as previously reported. $^{35}$

There are several limitations of our study. Firstly, the sample size is small and only 199 people were included in our study. Due to the low prevalence of CMBs, the power of the test was limited and further analysis on strictly lobar CMBs was ceased due to poor goodness-of-fit. Secondly, a semiquantitative method was used to assess white matter hyperintensity on MRI scans. However, the inter-rater reliability between the countries for MRI evaluation was excellent. $^{12}$ Thirdly, some other risk factors of CMBs were not included, such as dyslipidemia and inflammation markers (eg, CRP).

\section{Conclusion}

Our study found that multiple factors including hypertension, CSVD (lacunar infarction and white matter hyperintensities), and genetic factors (APOE and CR1) increased 
the risk of CMBs in a Chinese stroke-free non-demented community elderly. These findings might help to understand the mechanism underlying CMBs and prevent cerebrovascular disease in Chinese elderly.

\section{Ethics Approval}

The study was conducted in accordance with the Declaration of Helsinki and was approved by the Institutional Ethics Committee of Shanghai Jiao Tong University School of Medicine ((2011) ethics committee No.13).

\section{Consent to Participate}

Written informed consent was obtained from each participant of this study.

\section{Acknowledgments}

We thank all doctors from Wuliqiao Medical Center for their support with our epidemiology study.

\section{Author Contributions}

All authors made substantial contributions to conception and design, acquisition of data, or analysis and interpretation of data; took part in drafting the article or revising it critically for important intellectual content; agreed to submit to the current journal; gave final approval of the version to be published; and agree to be accountable for all aspects of the work.

\section{Funding}

This study was supported by grants from National Natural Science Fund (81571103).

\section{Disclosure}

The authors have no conflicts of interest to declare.

\section{References}

1. Haller S, Vernooij MW, Kuijer JPA, et al. Cerebral microbleeds: imaging and clinical significance. Radiology. 2018;287(1):11-28. doi:10.1148/radiol.2018170803

2. Charidimou A, Shams S, Romero JR, et al. Clinical significance of cerebral microbleeds on MRI: a comprehensive meta-analysis of risk of intracerebral hemorrhage, ischemic stroke, mortality, and dementia in cohort studies (v1). Int J Stroke. 2018;13(5):454-468. doi:10.1177/ 1747493017751931

3. Yakushiji Y, Wilson D, Ambler G, et al. Distribution of cerebral microbleeds in the East and West. Neurology. 2019;92(10):e1086e1097. doi:10.1212/WNL.0000000000007039

4. Hilal S, Mok V, Youn YC, et al. Prevalence, risk factors and consequences of cerebral small vessel diseases: data from three Asian countries. J Neurol Neurosurg Psychiatry. 2017;88(8):669-674. doi:10.1136/jnnp-2016-315324
5. Mok V, Ding D, Fu J, et al. Transcranial doppler ultrasound for screening cerebral small vessel disease. Stroke. 2012;43 (10):2791-2793. doi:10.1161/STROKEAHA.112.665711

6. Han F, Zhai FF, Wang Q, et al. Prevalence and risk factors of cerebral small vessel disease in a Chinese population-based sample. $J$ Stroke. 2018;20(2):239-246. doi:10.5853/jos.2017.02110

7. Vernooij MW, van der Lugt A, Ikram MA, et al. Prevalence and risk factors of cerebral microbleeds: the Rotterdam Scan Study. Neurology. 2008;70(14):1208-1214. doi:10.1212/01.wnl.00003077 50.41970.d9

8. Yubi T, Hata J, Ohara T, et al. Prevalence of and risk factors for cerebral microbleeds in a general Japanese elderly community. Neurol Clin Pract. 2018;8(3):223-231. doi:10.1212/CPJ.00000 00000000464

9. Ma JF, Xin XY, Liang L, et al. Restless legs syndrome in Chinese elderly people of an urban suburb in Shanghai: a community-based survey. Parkinsonism Relat Disord. 2012;18(3):294-298. doi:10.1016/j.parkreldis.2011.11.014

10. Ma JF, Qiao Y, Gao X, et al. A community-based study of risk factors for probable rapid eye movement sleep behavior disorder. Sleep Med. 2017;30:71-76. doi:10.1016/j.sleep.2016.06.027

11. Gregoire SM, Chaudhary UJ, Brown MM, et al. The Microbleed Anatomical Rating Scale (MARS): reliability of a tool to map brain microbleeds. Neurology. 2009;73(21):1759-1766. doi:10.1212/ WNL.0b013e3181c34a7d

12. Wahlund LO, Barkhof F, Fazekas F, et al. A new rating scale for age-related white matter changes applicable to MRI and CT. Stroke. 2001;32(6):1318-1322. doi:10.1161/01.STR.32.6.1318

13. Qi X, Tang H, Luo Q, et al. White matter hyperintensities predict cognitive decline: a community-based study. Can J Neurol Sci. 2019;46(4):383-388. doi:10.1017/cjn.2019.47

14. Wardlaw JM, Smith EE, Biessels GJ, et al. Neuroimaging standards for research into small vessel disease and its contribution to ageing and neurodegeneration. Lancet Neurol. 2013;12(8):822-838. doi:10.1016/S1474-4422(13)70124-8

15. Johansson JU, Brubaker WD, Javitz H, et al. Peripheral complement interactions with amyloid beta peptide in Alzheimer's disease: polymorphisms, structure, and function of complement receptor 1 . Alzheimers Dement. 2018;14(11):1438-1449. doi:10.1016/j. jalz.2018.04.003

16. Tsai -H-H, Pasi M, Tsai L-K, et al. Superficial cerebellar microbleeds and cerebral amyloid angiopathy. Stroke. 2020;51(1):202-208. doi:10.1161/STROKEAHA.119.026235

17. Poels MM, Vernooij MW, Ikram MA, et al. Prevalence and risk factors of cerebral microbleeds: an update of the Rotterdam scan study. Stroke. 2010;41(10 Suppl):S103. doi:10.1161/ STROKEAHA.110.595181

18. Rannikmae K, Samarasekera N, Martinez-Gonzalez NA, Al-Shahi Salman R, Sudlow CL. Genetics of cerebral amyloid angiopathy: systematic review and meta-analysis. $J$ Neurol Neurosurg Psychiatry. 2013;84(8):901-908. doi:10.1136/jnnp-2012-303898

19. Caunca MR, Del Brutto V, Gardener H, et al. Cerebral microbleeds, vascular risk factors, and magnetic resonance imaging markers: the northern Manhattan study. J Am Heart Assoc. 2016;5:9. doi:10.1161/ JAHA.116.003477

20. Li HQ, Cai WJ, Hou XH, et al. Genome-wide association study of cerebral microbleeds on MRI. Neurotox Res. 2020;37(1):146-155. doi:10.1007/s12640-019-00073-3

21. de Vries MA, Trompet S, Mooijaart SP, et al. Complement receptor 1 gene polymorphisms are associated with cardiovascular risk. Atherosclerosis. 2017;257:16-21. doi:10.1016/j.atherosclerosis.20 16.12.017

22. Boiocchi C, Zorzetto M, Sbarsi I, et al. CR1 genotype and haplotype involvement in coronary artery disease: the pivotal role of hypertension and dyslipidemia. Int $J$ Mol Med. 2009;24(2):181-187. doi:10.3892/ijmm_00000221 
23. Romero JR, Preis SR, Beiser A, et al. Carotid atherosclerosis and cerebral microbleeds: the Framingham heart study. J Am Heart Assoc. 2016;5(3):e002377. doi:10.1161/JAHA.115.002377

24. Mahley RW. Apolipoprotein E: from cardiovascular disease to neurodegenerative disorders. J Mol Med (Berl). 2016;94(7):739-746. doi:10.1007/s00109-016-1427-y

25. Klop B, van der Pol P, van Bruggen R, et al. Differential complement activation pathways promote $\mathrm{C} 3 \mathrm{~b}$ deposition on native and acetylated LDL thereby inducing lipoprotein binding to the complement receptor 1. J Biol Chem. 2014;289(51):35421-35430. doi:10.1074/jbc. M114.573840

26. Low A, Mak E, Rowe JB, Markus HS, O’Brien JT. Inflammation and cerebral small vessel disease: a systematic review. Ageing Res Rev. 2019;53:100916. doi:10.1016/j.arr.2019.100916

27. Dose J, Huebbe P, Nebel A, Rimbach G. APOE genotype and stress response - a mini review. Lipids Health Dis. 2016;15(1):121. doi:10.1186/s12944-016-0288-2

28. Tao Q, Ang TFA, DeCarli C, et al. Association of chronic low-grade inflammation with risk of alzheimer disease in apoe4 carriers. JAMA Netw Open. 2018;1(6):e183597. doi:10.1001/jamanetworkopen.2018.3597

29. Collard CD, Bukusoglu C, Agah A, et al. Hypoxia-induced expression of complement receptor type 1 (CR1, CD35) in human vascular endothelial cells. Am J Physiol. 1999;276(2):C450. doi:10.1152/ajpcell.1999.276.2.C450
30. Vlaicu SI, Tatomir A, Rus V, et al. The role of complement activation in atherogenesis: the first 40 years. Immunol Res. 2016;64(1):1-13. doi:10.1007/s12026-015-8669-6

31. Pantoni L. Cerebral small vessel disease: from pathogenesis and clinical characteristics to therapeutic challenges. Lancet Neurol. 2010;9(7):689-701. doi:10.1016/S1474-4422(10)70104-6

32. Qiu C, Sigurdsson S, Zhang Q, et al. Diabetes, markers of brain pathology and cognitive function: the age, gene/environment susceptibility-Reykjavik study. Ann Neurol. 2014;75(1):138-146. doi:10.1002/ana.24063

33. Wilson D, Werring DJ. Antithrombotic therapy in patients with cerebral microbleeds. Curr Opin Neurol. 2017;30(1):38-47. doi:10.1097/WCO.0000000000000411

34. Ge L, Ouyang X, Ban C, et al. Cerebral microbleeds in patients with ischemic cerebrovascular disease taking aspirin or clopidogrel. Medicine. 2019;98(9):e14685. doi:10.1097/MD.0000000000014685

35. Romero JR, Preis SR, Beiser A, et al. Risk factors, stroke prevention treatments, and prevalence of cerebral microbleeds in the Framingham Heart Study. Stroke. 2014;45(5):1492-1494. doi:10.1161/STROKEAHA.114.004130

\section{Publish your work in this journal}

Therapeutics and Clinical Risk Management is an international, peerreviewed journal of clinical therapeutics and risk management, focusing on concise rapid reporting of clinical studies in all therapeutic areas, outcomes, safety, and programs for the effective, safe, and sustained use of medicines. This journal is indexed on PubMed Central, CAS,
EMBase, Scopus and the Elsevier Bibliographic databases. The manuscript management system is completely online and includes a very quick and fair peer-review system, which is all easy to use. Visit http://www.dovepress.com/testimonials.php to read real quotes from published authors. 